\title{
Mineração
}

\section{Influência do estado de agregação da polpa na flotação de quartzo, apatita e calcita}

\author{
Marisa Martins \\ Doutoranda em Engenharia Mineral, Departamento de Engenharia de Minas e de Petróleo \\ Escola Politécnica, Universidade de São Paulo, São Paulo,Brasil.E-mail: marisa.martins@poli.usp.br \\ Laurindo de Salles Leal Filho \\ Professor Titular da Escola Politécnica da USP, Departamento de Engenharia de Minas e de Petróleo \\ Escola Politécnica, Universidade de São Paulo, São Paulo, Brasil.E-mail: lauleal@usp.br
}

Paulo Roberto Leite

Gerente de Supply Chain da ExxonMobil Quimica Ltda, São Paulo, Brasil.E-mail: paulo.leite@exxonmobil.com

José Renato Baptista de Lima

Professor da Escola Politécnica da USP, Departamento de Engenharia de Minas e de Petróleo Escola Politécnica, Universidade de São Paulo, São Paulo, Brasil.E-mail: jrblima@usp.br

\section{Resumo}

Muitos estudos acadêmicos foram realizados nos Estados Unidos, durante as décadas de 30 e 40, motivados pela correlação entre o estado de agregação da polpa e a recuperação do processo de flotação, observada no dia-a-dia das usinas. O objetivo desse trabalho é contribuir para uma maior compreensão do fenômeno observado na prática, dentro da realidade brasileira. Ensaios de microflotação e grau de dispersão foram realizados com partículas de quartzo, apatita e calcita. Os resultados reforçam a afirmativa postulada por Taggart e colaboradores de que a agregação ocorre como efeito da ação de reagentes e não como a causa de uma boa flotabilidade.

Palavras-chave: flotação, agregação e dispersão.

\begin{abstract}
Many academic studies were carried out in the United States during the 1930's and 1940's, motivated by the correlation between the pulp aggregation state and the recovery at the flotation process, observed in industrial circuits. The aim of this work is to contribute to a better understanding of the phenomenon within the Brazilian reality. Microflotation and dispersion tests were carried out with quartz, apatite and calcite particles. The results strengthen the assertion by Taggart and co-workers that the aggregation occurs in the pulp as an effect of the reagents action and not as the primary cause of good flotability.
\end{abstract}

Keywords: flotation, aggregation, dispersion. 


\section{Introdução}

Logo nos primórdios da aplicação industrial do processo de flotação, constatou-se, no dia-a-dia das usinas, que existia uma evidente correlação entre o estado de agregação da polpa e a recuperação do processo (Sulman,1919); (Taggart et al., 1934) e (Fitt et al., 1943). Como conseqüência disto, entre os anos 20 e 30 , circulava entre os operadores de usinas de flotação dos Estados Unidos um ditado muito interessante do ponto de vista teórico e prático: "to float a mineral, you must first flocculate it" (Taggart et al., 1934, P.367). Esse ditado motivou muitos estudos de cunho acadêmico nos Estados Unidos durante as décadas de 30 e 40, que resultaram em duas vertentes (Gaudin,1932);(Taggart et al., 1934) e (Fitt et al., 1943):

i. Um primeiro grupo buscava dar suporte científico ao fenômeno observado na prática, acreditando que a agregação era a causa de uma boa recuperação e, conseqüentemente, a escolha dos reagentes de flotação deveria ser feita de modo a privilegiar a agregação dos minerais que se desejava flotar e aumentar a dispersão dos minerais que não se desejava flotar.

ii.Um segundo grupo, do qual Taggart e colaboradores faziam parte, assumia uma postura cética diante do fenômeno, postulando que a agregação era apenas um efeito da interação entre minúsculas bolhas existentes no seio da polpa e partículas previamente hidrofobizadas pela ação do coletor.

No final dos anos 40, chegou-se a um consenso de que Taggart e colaboradores estavam corretos. O assunto, desse modo, tornou-se praticamente esquecido, sepultando uma constatação extremamente útil e relevante para o en- genheiro de processos. No Brasil, em meados dos anos 80, o assunto reapareceu em várias pesquisas realizadas na Universidade Federal de Minas Gerais, gerando, então, uma série de publicações em português relevantes sobre o tema e, desse modo, ressuscitando velhas indagações bastante pertinentes (Tabela 1).

O objetivo desse trabalho é contribuir para uma maior compreensão do fenômeno observado na prática, buscando verificar os limites de sua validade.

Segundo Parfitt (1969), o termo dispersão é usado para se definir o processo completo de incorporação de partículas sólidas (finamente divididas) a um meio líquido, de maneira tal que o produto final resulte num estado uniforme de distribuição. Por outro lado, o estado de agregação se refere à estabilidade termodinâmica da dispersão como tal, geralmente em função do tempo. Partículas

Tabela 1 - Estudos relacionando flotabilidade e agregação.

\begin{tabular}{c|c|c}
\hline Referência & Sistemas minerais/reagentes & Conclusões (*) \\
\hline Coelho, 1984 & Apatita/oleato de sódio & FcA \\
\hline Coelho, 1984 & Quartzo/amina & FcD \\
\hline Galery; Peres, 1986 & Willemita/amina & FcA \\
\hline Da Luz, 1988 & Calcita, apatita, dolomita/ oleato de \\
sódio & Fluorita e cassiterita & $\begin{array}{c}\text { da fluorita e dispersão da cassiterita. A } \\
\text { fluorita (mais agregada) flotou em maior } \\
\text { magnitude que a cassiterita. }\end{array}$ \\
\hline Salum; Assis e Leal 1986 & $\begin{array}{c}\text { Minérios de fosfato com diferentes } \\
\text { solubilidades e composição } \\
\text { Filho, 1992 }\end{array}$ & $\begin{array}{c}\text { Apatita: FcA (coletor), FñcA (depressor) e } \\
\text { FcA (coletor + depressor). }\end{array}$ \\
\hline Salum; Valente, 1995 & $\begin{array}{c}\text { Calcita e apatita/ amido e oleato de } \\
\text { sódio }\end{array}$ & $\begin{array}{c}\text { Calcita: FcA. } \\
\text { Apatita: FcA. }\end{array}$ \\
\hline
\end{tabular}

(*) FcA = máximos de flotabilidade coincidiram com máximos de agregação; FcD = máximos de flotabilidade coincidiram com máximos de dispersão e FñcA = máximos de flotabilidade não coincidiram com máximos de agregação. 
de dispersões instáveis tendem a se agregar, minimizando sua energia interfacial, e a se segregar do meio, minimizando sua energia potencial (Da Luz; Araujo, 1988).

O estado de agregação pode ser estimado ou inferido através de propriedades a ele associadas (turbidez da suspensão, velocidade de sedimentação) e quantificado pelo grau de dispersão/ agregação, que constitui uma medida relativa. Araujo e Galery (1987) apresentam e discutem diversas técnicas para a determinação do grau de dispersão/agregação de sólidos em suspensão aquosa.

A estabilidade de um sistema disperso pode ser minimizada através do aumento do grau de agregação entre as partículas. Para, efetivamente, se conseguir tal intento, pode-se fazer uso de reagentes químicos que, em virtude de sua natureza e modo de ação, irão determinar se a agregação ocorrerá por coagulação, floculação, aglomeração ou sinergia, entre quaisquer desses mecanismos.

\section{Procedimentos experimentais}

\subsection{Obtenção das amostras}

Os minerais apatita e calcita foram obtidos da purificação de amostra do minério calcítico padrão da jazida de $\mathrm{Ca}$ jati-SP, através de ensaios de afunda-flutua em líquidos densos (bromofórmio e iodeto de metileno). O quartzo foi preparado a partir de amostra de quartzito. Os três minerais foram cominuídos e peneirados de modo a gerar dois produtos: um mais grosseiro $(-150+44 \mu \mathrm{m})$, utilizado nos ensaios de flotabilidade, e outro mais fino $(-32 \mu \mathrm{m})$, utilizado nos ensaios de determinação do grau de dispersão e de medidas de ponto isoelétrico (PIE). Difração de raios $\mathrm{X}$ foi utilizada para certificar a pureza das amostras.

\subsection{Reagentes utilizados}

Utilizou-se, como coletor para quartzo, a eteramina Flotigan EDA, produzida pela Clariant. Para a flotação de apatita e calcita, utilizou-se oleato de sódio como coletor e tall-oil para os ensaios de dispersão desses minerais, ambos reagentes sendo carboxilatos. Soluções foram preparadas com água destilada, na concentração desejada para cada teste. Para correção de $\mathrm{pH}$, utilizaram-se os reagentes $\mathrm{NaOH}$ e HCl P.A. preparados a $10 \%$ p/v.

\subsection{Ensaios de flotabilidade}

Para determinação da flotabilidade do quartzo, executaram-se ensaios em célula de flotação Cimaq, com cuba de $1500 \mathrm{~mL}$, a $1100 \mathrm{rpm}$. Os ensaios eram conduzidos adicionando $300 \mathrm{~g}$ de amostra em 1200 mL de água potável, perfazendo uma porcentagem de sólidos da ordem de 20\% em massa. Após correção do pH da polpa, para os valores desejados, condicionava-se esta com o coletor por 2 min, quando, então, se admitia ar no sistema, coletando-se a espuma até a exaustão.

A flotabilidade dos minerais apatita e calcita foi determinada através de ensaios de microflotação em tubo de Hallimond Modificado (placa porosa média-fina) em pH 10,3, sob as seguintes condições: $1 \mathrm{~g}$ do mineral puro era condicionado com solução de amido na concentração desejada por 2 min, seguido pelo condicionamento com oleato de sódio (12 mg/L) por $1 \mathrm{~min}$. O nível da solução era mantido constante (160 mL). Após condicionamento, flotação foi realizada durante o período de $1 \mathrm{~min}$ com uma vazão de gás nitrogênio de $60 \mathrm{~mL} / \mathrm{min}$.

Os produtos flutuado e afundado eram filtrados, secados e pesados. A flotabilidade foi assumida como sendo a razão entre a massa do produto flotado e a da alimentação, multiplicado por 100.

\subsection{Determinação do grau de dispersão}

Os graus de dispersão de suspensões dos minerais quartzo, apatita e calcita foram determinados segundo o método da sedimentação em proveta. Utilizou-se proveta de 100 mL com diâmetro de $30 \mathrm{~mm}$ acoplada a um tubo de borracha posicionado em sua porção inferior, cuja saída era aberta ou fechada através de uma pinça de Mohr. O modus operandi das determinações compreendia a adição de $1,0 \mathrm{~g}$ do mineral puro em 80 mL de solução, previamente preparada, para as condições que se desejava ( $\mathrm{pH}$ e concentração de reagentes), acompanhado de 1 min. de condicionamento, após o qual a proveta era virada de cabeça para baixo por 5 vezes, deixando o material decantar por 3 segundos, quando então se recolhia o material disperso através da abertura da pinça de Mohr. O material disperso e o sedimentado (agregado) eram secados e pesados. O grau de dispersão era calculado pela razão entre a massa de sólido dispersa após terem transcorrido 3 segundos de sedimentação e a massa total alimentada, multiplicada por 100.

\subsection{Determinação do Ponto Isoelétrico (PIE)}

O ponto isoelétrico do quartzo foi determinado através do método de $\mathrm{Mu}$ lar-Roberts (Mular; Roberts, 1966), baseado em mudanças do $\mathrm{pH}$ de suspensões, após variação da concentração de um eletrólito indiferente, utilizado para ocasionar variação da força iônica do sistema. $\mathrm{O} \mathrm{pH}$ inicial $\left(\mathrm{pH}_{\mathrm{i}}\right)$ da suspensão de 1,0 grama do mineral puro em $70 \mathrm{~mL}$ de solução $10^{-2} \mathrm{~N}$ de $\mathrm{KNO}_{3}$ era ajustado com $\mathrm{NaOH}$ ou $\mathrm{HCl} 10 \%$ p/v e o valor era anotado. Em seguida, adicionava-se $\mathrm{KNO}_{3}$ sob agitação, aumentando sua concentração de $10^{-2}$ para $10^{-1} \mathrm{~N}$. Mediase o $\mathrm{pH}$ final $\left(\mathrm{pH}_{\mathrm{f}}\right)$ após 10 segundos de repouso do sistema com $\mathrm{pH}$-metro $\mathrm{Mi}$ cronal, modelo B374. Calculava-se a diferença $\mathrm{pH}_{\mathrm{f}}-\mathrm{pH}_{\mathrm{i}}(\Delta \mathrm{pH})$ e traçava-se a curva de $\mathrm{pH}$ versus $\Delta \mathrm{pH}$.

\section{Resultados e discussão}

\subsection{Sistema quartzol eteramina}

A flotabilidade do quartzo com ete- 
ramina, em função do $\mathrm{pH}$, pode ser observada na Figura 1, onde se verifica um patamar de alta flotabilidade entre $\mathrm{pH}=5$ e pH=10. Já o grau de dispersão, em função do pH, é apresentado na Figura 2, onde é possível observar duas regiões de máxima dispersão: uma em $\mathrm{pH}=2,5$ e outra em $\mathrm{pH}=13$.

Comparando os resultados, observa-se uma correlação direta entre flotabilidade e agregação. O máximo de dispersão da polpa, em $\mathrm{pH}=2,5$ pode ser explicado pelo fato de que, em $\mathrm{pH}=2$, ocorre reversão do sinal de carga elétrica superficial do mineral (Figura 3). Como o coletor é catiônico e interage com o mineral, na forma de contra-íon na dupla camada elétrica, tal comportamento é esperado e corroborado pelo mínimo de flotabilidade observado no mesmo $\mathrm{pH}$.

O máximo de dispersão observado em $\mathrm{pH}=13$ pode estar associado à química do coletor em solução. Sendo o coletor uma eteramina, a partir de $\mathrm{pH}=10,5$ começam a ocorrer a diminuição da concentração da espécie ionizada (coletora) e um aumento da não ionizada. Tal comportamento foi corroborado pela queda de flotabilidade do quartzo, indicando uma menor interação mineral/coletor.

\subsection{Sistemas apatita/carboxilatos e calcital carboxilatos}

Realizaram-se medições do grau de dispersão dos minerais puros apatita e calcita com água destilada e $\mathrm{pH}=10,3$; na ausência de reagentes, na presença de tall-oil e na presença simultânea de amido e tall-oil, chegou-se aos resultados apresentados na Figura 4, onde é importante destacar que:

i. Nas três condições testadas, a apatita apresentou-se menos dispersa que a calcita.

ii.O tall-oil foi capaz de diminuir o grau de dispersão dos dois minerais em pequena monta.

iii. A ação conjunta da dupla amido/tall-oil resultou numa drástica queda do grau de dispersão de ambos minerais.

Os resultados dos ensaios de microflotação em tubo de Hallimond, para os minerais calcita e apatita, encontram-se expostos na Figura 5, onde se verifica que:

i. A apatita apresentou maior flotabilidade que a calcita, em todas as condições avaliadas.

ii.Ambos minerais sofreram um drástico aumento em flotabilidade, quando os reagentes foram condicionados em conjunto, indicando uma possível ação sinergética entre a dupla amido/ oleato.

Comparando os resultados apresentados pelos ensaios de dispersão e microflotação, tem-se que:

i. A apatita apresentou valores de grau de agregação e de flotabilidade maiores que os da calcita, ou seja, o mineral que apresentou a maior agregação foi também aquele com maior flotabilidade.



Figura 1 - Flotabilidade do quartzo com eteramina (250 g/t) versus $\mathrm{pH}$.

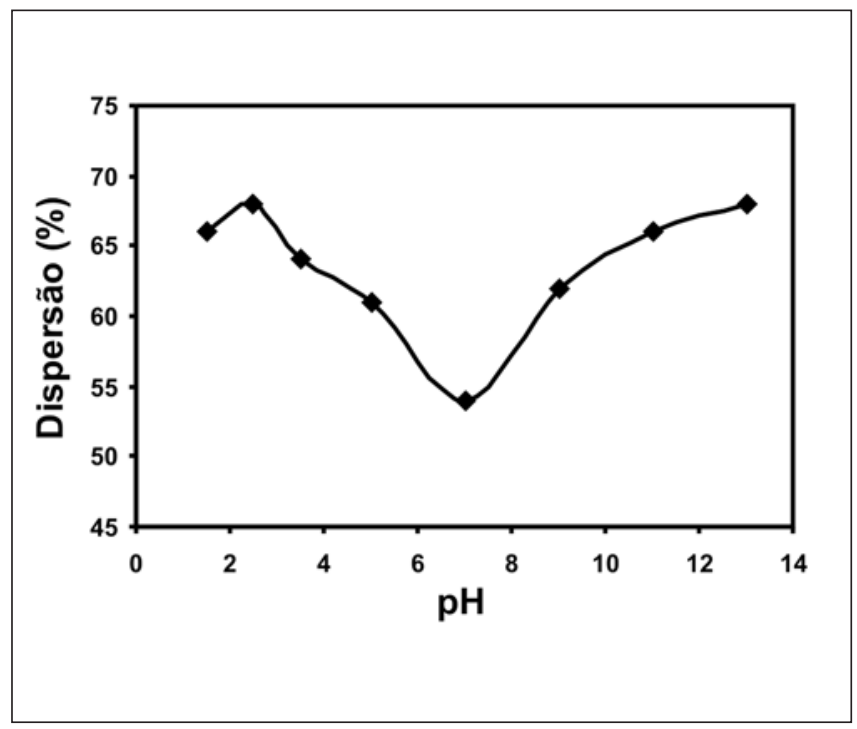

Figura 2 - Grau de dispersão do quartzo com eteramina (9mg/L) versus $\mathrm{pH}$.

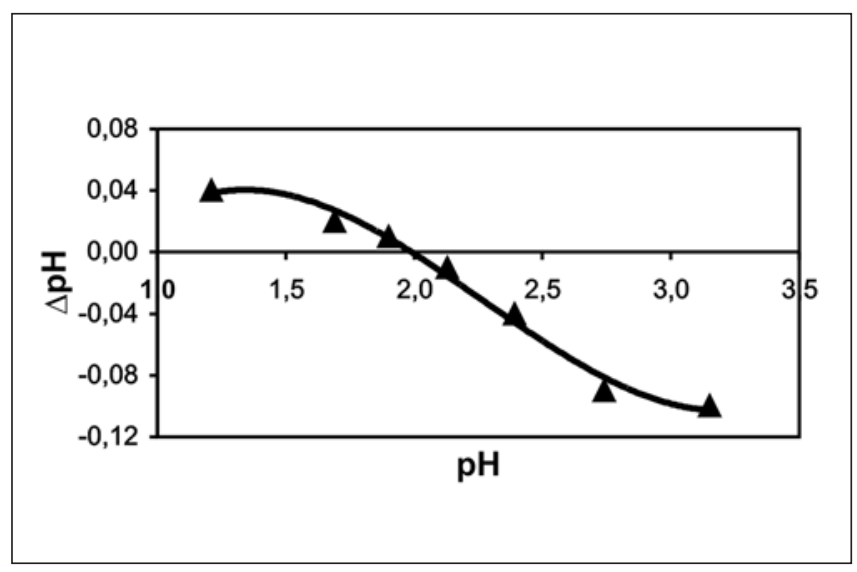

Figura 3 - PIE do quartzo pelo método de Mullar-Roberts. 
ii.Para ambos minerais, observa-se que o condicionamento destes com amido + coletor resultou em um grau de agregação maior que o condicionamento com o coletor apenas. Entretanto essa segunda condição foi a que apresentou maiores valores de flotabilidade.

Com base nesses resultados, acredita-se que o amido, por ser uma grande molécula polimérica, esteja agindo como um floculante, aumentando a agregação da polpa. Por outro lado, a existência de vários grupos $\mathrm{OH}$, em suas moléculas, permite sua adsorção na interface calcita/solução e/ou apatita/solução através de interações (complexação) entre tais grupos $\mathrm{OH}$ e sítios $\mathrm{CaOH}$ existentes na interface mineral/solução (Raju et al., 1997). Tal adsorção, certamente, seria responsável pela diminuição da flotabilidade dos minerais calcita e apatita verificada na Figura 5, na medida em que se aumenta a concentração de amido em solução.

\section{Conclusões}

Os resultados obtidos na presente investigação reforçam a afirmativa de Taggart de que a agregação acontece na polpa como efeito da ação de reagentes e não como a causa de uma alta flotabilidade:

1. No sistema quartzo/eteramina, observa-se uma relação direta entre agregação e flotabilidade. Entretanto o máximo e mínimos de flotabilidade se devem a condições favoráveis ou não de interação quartzo/coletor e não à agregação dos minerais. Esta poderia apenas fornecer um prognóstico, por ser apenas um efeito da interação do reagente com o mineral.

2. Nos sistemas apatita e calcita/carboxilatos, a maior agregação apresentada pela apatita frente à calcita coincidiu com a maior flotabilidade obtida pela apatita. Por outro lado, observou-se que os maiores valores de agregação, obtidos no condicionamento amido + coletor frente ao condicionamento com o coletor apenas, não coincidiram com maiores valores de flotabilidade.

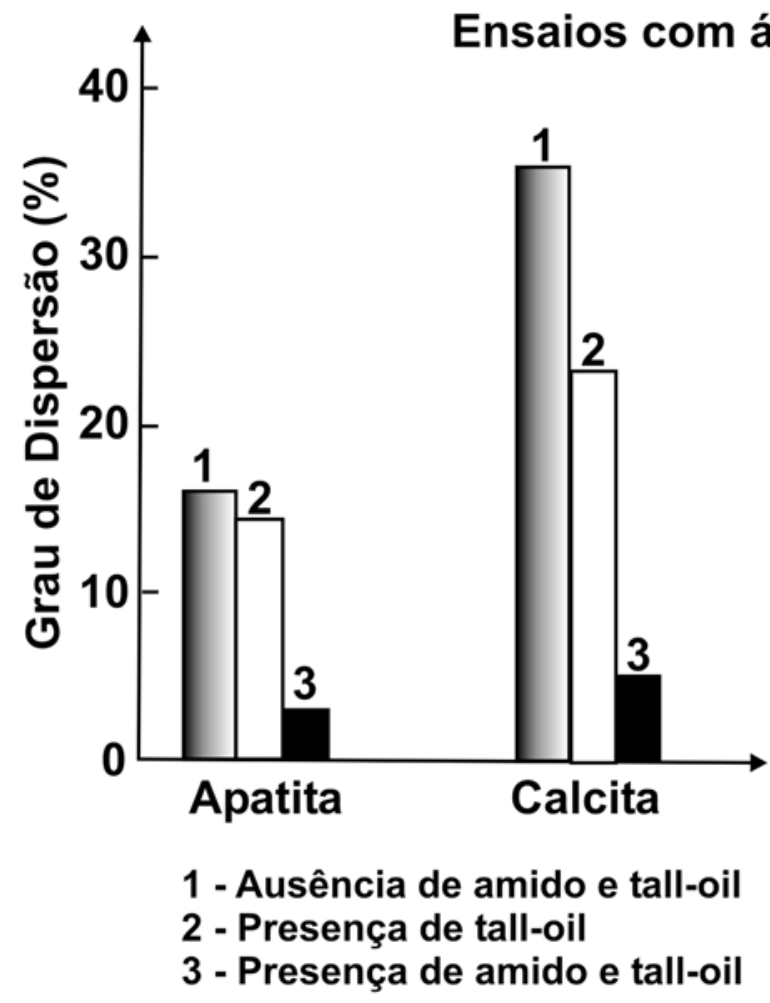

Figura 4 - Grau de dispersão dos minerais apatita e calcita na presença de água destilada, amido e tall-oil.

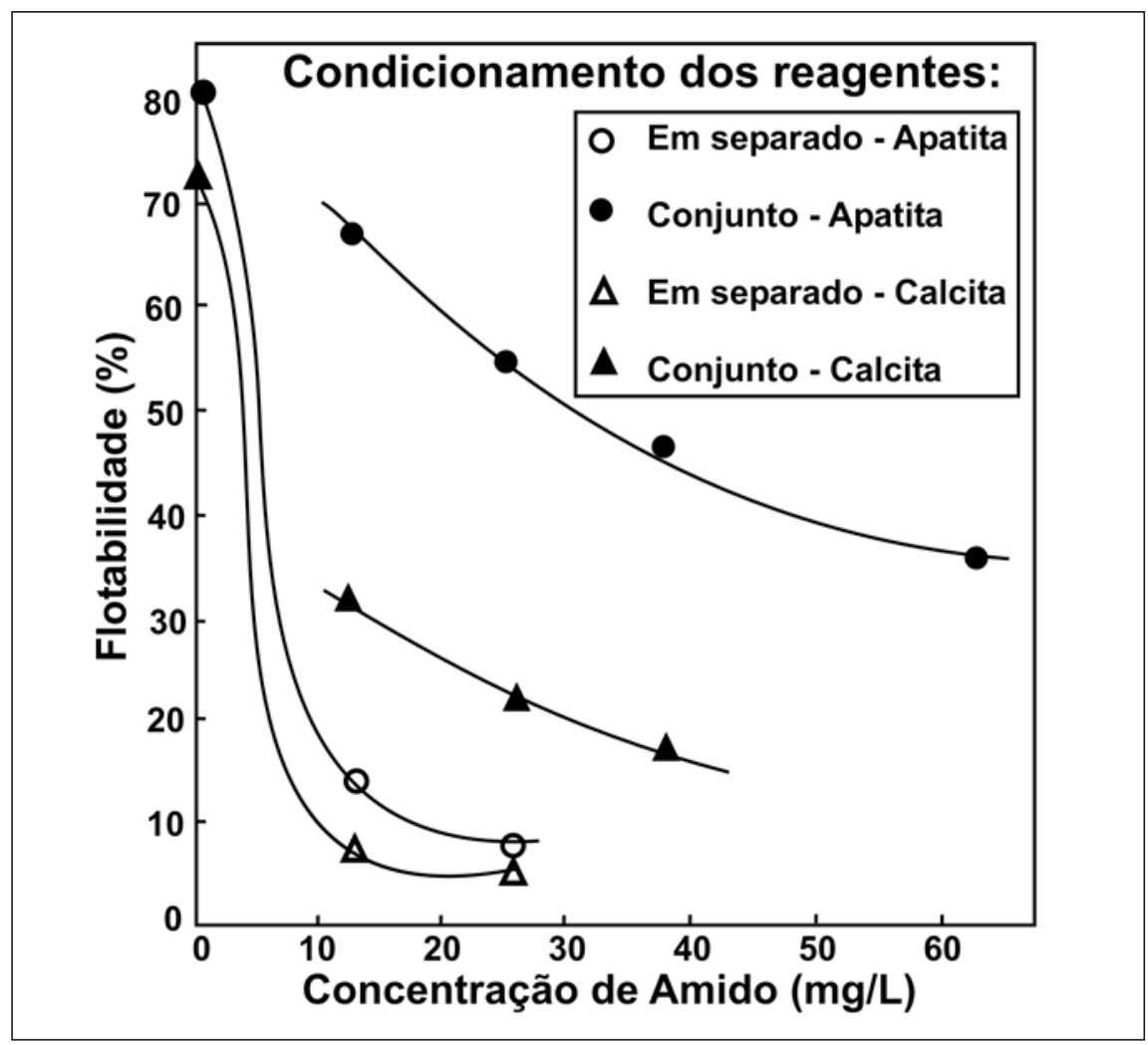

Figura 5 - Flotabilidade dos minerais apatita e calcita com oleato (12 mg/L) versus concentração de amido $(\mathrm{pH}=10,3)$. 


\section{Agradecimentos}

Os autores agradecem à FAPESP pelo financiamento do projeto $n^{\circ} 92 /$ 1550-6.

\section{Referências bibliográficas}

ARAUJO, A.C., GALERY, R. Técnicas para analisar dispersão/agregação. In: ENCONTRO NACIONAL DE TRATAMENTO DE MINÉRIOS E HIDROMETALURGIA, 12. Rio de Janeiro, 1987. Anais... S.n.t. v.1, p.23448.

COELHO, E.M. Separação de minerais de rochas fosfáticas e de minérios de ferro por flotação e por agregação seletiva. Belo Horizonte: Escola de Engenharia, Universidade Federal de Minas Gerais, 1984. 232p. (Tese Titular).

DA LUZ, J.A.M., ARAUJO, A.C. Fenômenos de agregação: sua influência sobre operações de processamento de minerais. In: ENCONTRO NACIONAL DE TRATAMENTO DE MINÉRIOS E HIDROMETALURGIA, 13. São Paulo, 1988. Anais... São Paulo: ABM/ APEMI/EPUSP, 1988. v.1, p.37-49
DA LUZ, J.A.M. Efeito do estado de agregação na separação entre calcita, dolomita e apatita por flotação. Belo Horizonte: Escola de Engenharia, Universidade Federal de Minas Gerais, 1988. 189p. (Tese de Mestrado).

FITT, T.C.,; THOMAS, A.W., TAGGART, A.F. The nature of dispersed mineral in flotation pulps. Transactions of the American Institute of Mining and Metallurgical Engineers, v.153, p. 49399, 1943.

GALERY, R., PERES, A.E.C. Estudo de dispersão no sistema Willemita/dolomita. Metalurgia. ABM, v.42, n.340, p.153-6, 1986.

GAUDIN, A.M. Flotation. New York: McGraw-Hill, 1932. p.358-85.

MULAR, A.L., ROBERTS, R.B. A simplified method to determine isoeletric points of oxides. Transactions of the Canadian Institute of Mining and Metallurgy, p.438-9, 1966.

OLIVEIRA, J.F. Flotação de cassiterita: um estudo de seletividade e alguns aspectos cinéticos. Rio de Janeiro: Universidade Federal do Rio de Janeiro, 1986. 142p. (Tese de Doutorado).

PARFITT, D.G. fundamental aspects of dispersion. In: PARFITT, G.D. (ed.) Dispersion of powders in liquids: with special reference to pigments. Amsterdam, Elsevier, 1969. Cap.3, p.81-121.

RAJU, B.G., HOLMGREN, A., FORSLING, W. Adsorption of dextrin at mineral/water interface. Journal of Colloid and Interface Science. New York, v.193, p.215-222, 1997.

SALUM, M.J.G., ASSIS, S.M., LEAL FILHO, L.S. Contribuição ao entendimento da relação entre agregação e desempenho na flotação. In: ENCONTRO DO HEMISFÉRIO SUL SOBRE TECNOLOGIA MINERAL, 3. Anais... S. Lourenço: 1992. p.116-135.

SALUM, M.J.G., VALENTE, D.A.L. Efeito agregante e depressor do amido no sistema mineral calcita/apatita. In: ENCONTRO NACIONAL DE TRATAMENTO DE MINÉRIOS E HIDROMETALURGIA, 16. Rio de Janeiro: 1995. Anais... S.n.t. v.1, p.239-48.

SULMAN, H.L. A contribution to the study of flotation. Bulletin - Institution of Mining and Me tallurgy.1919. $95 \mathrm{p}$.

TAGGART, A.F., DEL GIUDICE, G.R.M., ZIEHL, O.A. The case for the chemical theory of flotation. Transactions of the American Institute of Mining and Metallurgical Engineers. v. 112, p. 348381, 1934.

Artigo recebido em 01/08/2005 e aprovado em 11/01/2006.

\title{
REM - Revista Escola de Minas 70 anos divulgando CIÊNCIA.
}

$\star \star * \star * *$

\section{REM: a mais antiga revista técnico- científica do setor mínero-metalúrgico.}

\author{
$\star * \star * * *$
}

www.rem.com.br

$* * * * * *$ 